\section{Bioprinting for osteosarcoma model: Methodological aspects and experimental applications}

Elena Delgrosso, ${ }^{1}$ Gaia Martina

Sarcinelli,1 Laura Cansolino,1,6 Federica

Riva, ${ }^{3}$ Franca Scocozza, ${ }^{4}$

Michele Conti, ${ }^{4}$ Ian Postuma, 6

Silva Bortolussi,5,6 Cinzia Ferrari1,2,6

1Department of Clinical Surgical

Sciences; ${ }^{2}$ Animal Care and

Radiobiology Centre; 3Department of

Public Health, Experimental and

Forensic Medicine, Histology and

Embryology Unit; ${ }^{4}$ Department of Civil

Engineering and Architecture;

5Department of Physics University of

Pavia, Pavia; ${ }^{\text {National Institute of }}$

Nuclear Physics, Pavia section, Pavia, Italy

\begin{abstract}
The study aims at using the bioprinting technique to create an in vitro $3 \mathrm{D}$ construct of osteosarcoma, as an alternative model for studies related to Boron Neutron Capture Therapy (BNCT).
\end{abstract}

\section{Introduction}

Osteosarcoma is the most common primary malignant bone tumor affecting children and adolescents. Despite the introduction of several therapeutic options, the treatment and cure of osteosarcoma still remains an open challenge, due to its infiltrative growth that leads to a high incidence of metastasis and, therefore, to low survival rates. For this reason, Boron Neutron Capture Therapy (BNCT) has been investigated as an alternative or integrative treatment. BNCT is an experimental binary radiotherapy based on the irradiation with low energy neutron of neoplastic cells previously enriched with atoms of 10-boron (10B). ${ }^{1}$ This alternative technique selectively destroys neoplastic cells while sparing normal ones. To date, experimentation has been conducted on both in vitro and in vivo models, but animal testing has several disadvantages. In recent years, 3D bioprinting has led to rapid progress towards the modelling of pathological tissues. In particular, this technique is attracting for the engineering of tumor tissues, as it allows to mimic in vitro the tumor microenvironment thus improving the ability in modelling cancer compared to the existing methods. ${ }^{2}$ In this context, the goal of proposed work is to create $3 \mathrm{D}$ in vitro tumor models for BNCT studies. In particular, this study focuses on the optimization of the technical protocol for bioprinting the in vitro model in terms of construct geometry, hydrogel composition, cell density, crosslinking frequency and boron uptake.

\section{Materials and Methods}

Rat osteosarcoma cell line (UMR-106) grow adherent in DMEM medium (Dulbecco's modified Eagles Medium, Lonza) supplemented with $10 \%$ of fetal bovine serum (FBS) (Euroclone) and 1\% gentamicin (Euroclone), at $37^{\circ} \mathrm{C}$ in a humidified $5 \% \mathrm{CO}_{2}$ atmosphere.

The following printing protocol is the result of a series of tests carried out with the parameters summarized in Table 1. At about $80 \%$ of confluence cells were encapsulated into a sodium alginate-SA $8 \%$ and gelatinGL 4\% (Sigma Aldrich) hydrogel and 3D bioprinted with the Cellink INKREDI$\mathrm{BLE}^{\circledR}{ }^{\circledR}($ Cellink $\mathrm{AB})$ in order to obtain constructs with $2 \times 10^{6}$ cells. After printing, 3D constructs were crosslinked for 5 minutes with $2 \%$ Calcium Chloride- $\mathrm{CaCl}_{2}$ (Sigma Aldrich) and cultured in DMEM.

Construct colonization - At 0, 5, 7, 14, 21,28 , and 35 days constructs were fixed with ethanol $70 \%$, followed by Hoechst staining (HO) by observation with a fluorescence microscope.

Intracellular boron evaluation - To quantify the intracellular levels of boron, constructs with a concentration of $4 \times 10^{6}$ cells were printed, to mimic the quantity of cells contained in samples usually used in 2D studies for the evaluation of the boron concentration by neutron autoradiography.
Correspondence: Elena Delgrosso, Department of Clinical Surgical Sciences University of Pavia, Pavia, Italy.

E-mail: elena.delgrosso01@universitadipavia.it

Key words: 3D bioprinting; osteosarcoma in vitro model; 3D cell culture; BNCT.

Disclosures: The authors declare no conflict of interest.

Conference presentation: This paper was presented at the Third Centro 3R Annual Meeting - L'era delle 3R: modelli in silico, in vitro e in vivo per promuovere la ricerca traslazionale 30 September - 1 October 2021, Evento online organizzato dal Politecnico di Torino.

Received for publication: 9 July 2021.

Accepted for publication: 7 September 2021.

This work is licensed under a Creative Commons Attribution NonCommercial 4.0 License (CC BY-NC 4.0).

(C) Copyright: the Author(s), 2021

Two different treatments were tested: constructs printed with cells previously exposed to Boronophenylanine (BPA) 80 ppm for 4 hours (pre-printing treatment) and constructs printed with cells not previously enriched with BPA, but exposed after printing to BPA at a concentration of 80 $\mathrm{ppm} / 4 \mathrm{~h}$ (post-printing treatment). The preprinting treatment was intended to evaluate the gel interference with the ${ }^{10} \mathrm{~B}$ quantification. The post-printing one was aimed to verify the gel influence on the intracellular boron uptake and to define a method suitable to remove the residual BPA trapped in the gel matrix at the end of the contact time. To this end constructs were washed three times with PBS or DMEM solutions for 10', $15^{\prime}$ or $30^{\prime}$ (Table 1$)$.

\begin{tabular}{|c|c|c|c|}
\hline \multicolumn{4}{|c|}{ PRINTING PARAMETERS } \\
\hline Hydrogel & Cell concentration & Geometry & Crosslinking \\
\hline SA $6 \%-G L 4 \%$ & $1 \times 10^{6}$ cells/construct & full circumference & $\begin{array}{l}\mathrm{CaCl}_{2} \text { for } 5 \text { minutes } \\
\text { every week }\end{array}$ \\
\hline SA $8 \%$-GL $4 \%$ & $2 \times 10^{6}$ cells $/$ construct & circumference with grid & $\begin{array}{c}\mathrm{CaCl}_{2} \text { for } 5 \text { minutes } \\
\text { every two weeks }\end{array}$ \\
\hline \multicolumn{4}{|c|}{ BORON UPTAKE } \\
\hline \multicolumn{4}{|c|}{ PRE-PRINTING TREATMENT } \\
\hline Cell concentration & BPA treatment & \multicolumn{2}{|c|}{ Washing treatment } \\
\hline $4 \times 10^{6}$ cells $/$ construct & $80 \mathrm{ppm} / 4 \mathrm{~h}$ & \multicolumn{2}{|c|}{ Three times with PBS } \\
\hline \multicolumn{4}{|c|}{ POST- PRINTING TREATMENT } \\
\hline Cell concentration & BPA treatment & \multicolumn{2}{|c|}{ Washing treatment } \\
\hline \multirow{6}{*}{$4 \times 10^{6}$ cells $/$ construct } & \multirow{6}{*}{$80 \mathrm{ppm} / 4 \mathrm{~h}$} & \multirow{3}{*}{ PBS } & $3 \times 10^{\prime}$ \\
\hline & & & $3 \times 15^{\prime}$ \\
\hline & & & $3 \times 30^{\prime}$ \\
\hline & & \multirow{3}{*}{ DMEM } & $3 \times 10^{\prime}$ \\
\hline & & & $3 \times 15^{\prime}$ \\
\hline & & & $3 \times 30^{\prime}$ \\
\hline
\end{tabular}

Table 1. Summary of the experiments for the optimization of the technical protocol and the boron uptake. 


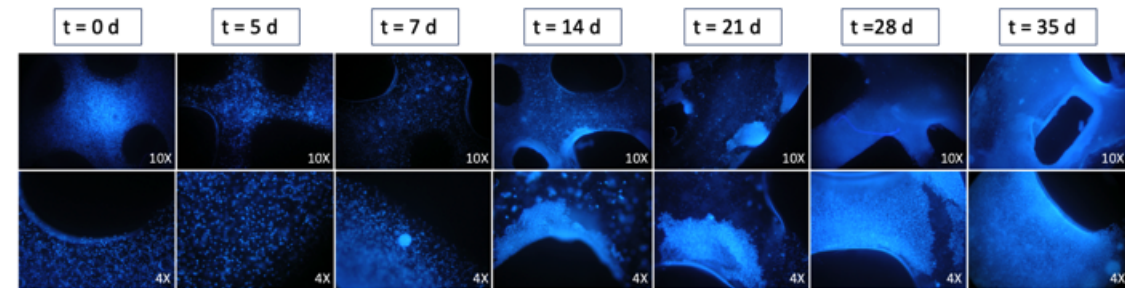

Figure 1. Microscopical observations at different end points of the 3D structures printed with an initial concentration of $2 \times 10^{6}$ cells/constructs. Nuclei stained by Hoechst 33258 , blue fluorescence.

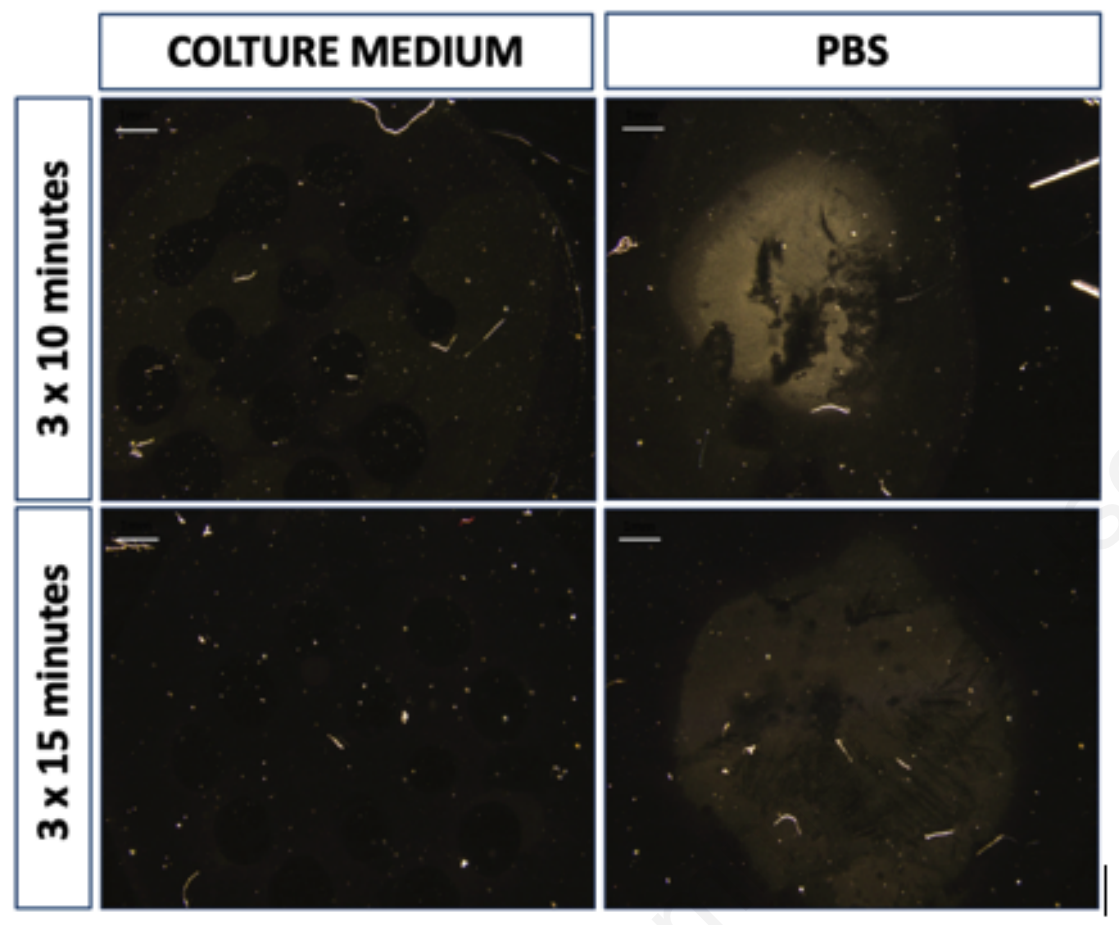

Figure 2. Qualitative neutron autoradiography images of boron biodistribution in 3D cellular constructs. Comparison between PBS and DMEM washed samples.

\section{Results}

Construct colonization - Microscopic observations at different end points of the $3 \mathrm{D}$ printed structures evidenced the pres-
Intracellular boron evaluation Preliminary results obtained from the preprinting condition showed that the hydrogel interferes with the intracellular boron measurement. In fact, traces generated by the neutron capture reaction are dispersed in the thickness of the gel, limiting the actual quantification of the signal by about $70 \%$, compared to values obtained in the correspondent 2D samples. With regard to the construct washing procedure 15 ' wash with DMEM was found to be suitable for removing the boron background (Figure 2).

\section{Discussion and Conclusions}

3D bioprinted osteosarcoma constructs should represent an effective alternative model in the context of the 3Rs principle (Replacement, Reduction, Refinement) to reduce the use of animal models for BNCT studies. The intracellular boron evaluation is a crucial point for BNCT studies. The method for its quantification in cells within constructs needs to be further refined. These preliminary studies have allowed us to obtain important information for its development. We expect that by improving the method it will be possible to use this newly 3D osteosarcoma model for BNCT studies.

\section{References}

1. Barth RF, Coderre JA, Vicente MG, Blue TE. Boron neutron capture therapy of cancer: current status and future prospects. Clin Cancer Res 2005; 11:3987-4002.

2. Bahcecioglu G, Basara G, Ellis BW, et al. Breast cancer models: Engineering the tumor microenvironment. Acta Biomater 2020;106:1-21. 